\title{
The Effects of Changing Chemistry on the Shock Response of Basic Polymers
}

\author{
J. C. F. Millett ${ }^{1}$ E. N. Brown ${ }^{2}$ - G. T. Gray $\mathrm{III}^{2} \cdot$ N. K. Bourne ${ }^{3}$. \\ D. C. Wood $^{4} \cdot$ G. Appleby-Thomas ${ }^{4}$
}

Received: 3 March 2016/ Accepted: 26 April 2016/Published online: 5 May 2016

(C) Crown Copyright 2016

\begin{abstract}
The shock response of four common semicrystalline thermoplastic polymers-polyethylene (PE), polyvinylchloride (PVC), polytetrafluoroethylene (PTFE) and polychlorotrifluoroethylene (PCTFE) - have been studied in terms of their Hugoniots, release velocities and shear strengths. Through the variations in behaviour caused by changes to the attached atoms to the carbon backbone, it has been possible to suggest that there are two main factors in play. The first is an electrostatic repulsion between adjacent polymer chains. Where this force is large, for example in PTFE with highly electronegative fluorine atoms, this results in this force dominating the shock response, with low shock velocities, high release velocities and little if no hardening behind the shock front. In contrast, in materials such as PE, this force is now weaker, due to the lower electronegativity of hydrogen, and hence this force is easier to overcome by the applied shock stress. Now the main factor affecting shock behaviour is controlled by the shape of the polymer chain allowing inter chain tangling (tacticity). This results in higher shock velocities, lower release speeds and significant hardening behind the shock front as the chains are forced together. This is prevalent in materials with a
\end{abstract}

J. C. F. Millett

jeremy.millett@awe.co.uk

1 AWE, Aldermaston, Reading RG7 4PR, UK

2 Los Alamos National Laboratory, Los Alamos, NM 87545, USA

3 Department of Materials Science, University of Manchester, Manchester, UK

4 Centre for Defence Engineering, Cranfield University, Defence Academy of the United Kingdom, Shrivenham, Swindon SN6 8LA, UK relatively open structure such as PE and is enhanced with the presence of large side groups or atoms off the main polymer chain.

Keywords Polyethylene · Polyvinylchloride ·

Polytetrafluoroethylene · Polychlorotrifluoroethylene .

Shock

\section{Introduction}

Interest in the response of polymeric materials to high strain-rate and shock loading conditions is driven by a number of factors, chief amongst them is their role as binder phases in energetic materials [1-4] and inert structural composite systems [5-7]. In addition, materials such as neoprene have found application in earthquake protection of buildings [8]. Detailed programmes on the shock response of polymers have until recently been relatively sparse when compared to other materials. Polymethylmethacrylate (PMMA), an amorphous transparent thermoplastic also know as acrylic, Plexiglas, Lucite, or Perspex, has received a deal of attention due to its use as a window material for interferometric measurements [9], whilst thermosetting epoxy based resins have been studied as they are often used as adhesives in the manufacture of target assemblies [10-12]. However, it should be appreciated that polymers have an extremely wide range of structural variation at the molecular level, and thus it is often difficult to identify trends and common features that describe their response to shock loading. Despite this, some common features have been identified; for example in plotting the shock velocity $\left(U_{\mathrm{S}}\right)$ against particle velocity $\left(u_{\mathrm{p}}\right)$, whilst the majority of polymers display a linear response of the form, 
$U_{S}=c_{0}+S u_{p}$

where $c_{0}$ and $S$ are empirically derived constants [13], it has been observed that unlike simple metals, the value of $c_{0}$ does not equate with the measured value of bulk sound speed $\left(c_{\mathrm{B}}\right)$, but rather is significantly higher. Indeed in many polymers it was observed that it lay above the ambient pressure longitudinal sound speed $\left(c_{\mathrm{L}}\right)$ [11, 1416]. In some polymers such as PMMA [9] and polyvinylidene difluoride (PVDF) [17], a non-linear $U_{\mathrm{S}}-u_{\mathrm{p}}$ response, with shock velocity dropping at lower particle velocities was observed. It has been subsequently suggested that this may be a more typical polymeric response [18], although rarely observed since the minimum particle velocity measured may have been above the non-linear part of the Hugoniot. Particulate composites with a hard particle in a soft binder, for example glass beads in hydroxyterminated polybutadiene (HTPB) [19] or the explosive RDX, also in HTPB $[1,20]$ have been observed to behave in a similar way, prompting Bourne and Milne [20] to suggest that this was due to a transition from elastic to plastic behaviour in the hard particles. Bourne et al. [18] went on to suggest that as many polymers are semi-crystalline in nature, a similar effect was taking place between the crystalline and amorphous regions within the polymer microstructure. In-situ neutron diffraction measurements of the elastic moduli in polytetrafluoroethylene (PTFE) [21] indicated that the modulus along the carbon-carbon backbone was of the order $220 \mathrm{GPa}$, whilst in the amorphous phase it was much lower at ca. $0.38 \mathrm{GPa}$. Another feature of the shared shock behaviour of polymers lies in a change in slope of the $U_{\mathrm{S}}-u_{\mathrm{p}}$ curve above $20 \mathrm{GPa}$ (precise values varying from polymer to polymer). Carter and Marsh [14] suggested that this be due to a re-ordering of the bonding within the polymer molecules from a predominantly two dimensional configuration to a more three dimensional situation due to the breaking and reformation of carboncarbon bonds.

As stated above, the large variation in molecular chemistry displayed by polymer molecules can make a systematic investigation somewhat difficult. However, matters can be simplified somewhat if a molecular feature is 'fixed', for example the carbon-carbon backbone. In a previous article [15], the complexity of the monomer unit was systematically increased by changing the nature of a single side group, starting with polyethylene (PE), moving to polypropylene (PP) with a dangling methyl side group and finally to polystyrene (PS) with a dangling benzene ring. It was observed that the Hugoniots in longitudinal stress $\left(\sigma_{\mathrm{x}}\right)$ particle velocity space increased from PE to PS, but more significantly the differences between the calculated hydrodynamic pressure $\left(P_{\mathrm{HD}}\right)$ where,
$P_{H D}=\rho_{0} U_{S} u_{p}$

with $\rho_{0}$ the ambient density, and the measured longitudinal stress. As this is defined in terms of the hydrostatic pressure $(P)$ and the shear strength $(\tau)$, via,

$\sigma_{x}=P+\frac{4}{3} \tau$,

this is an indication that the shear strength is increasing with pressure. Further, at a fixed particle velocity of $0.8 \mathrm{~mm} \mathrm{\mu s}^{-1}$, this difference was seen to increase from PE to PP, with PS being the largest, corresponding to an increase in size of the dangling side group. This was later confirmed by direct measurement of the shear strength in the three materials [22] where it was suggested that increasing the size of the side group increased the degree of tangling between adjacent polymer chains, and hence increase the forces required to move those chains closer.

In a similar series of experiments, the effects of fluorine additions to the basic polyethylene molecule were investigated, using the materials polyethylene, polyvinylidene difluoride (PVDF-two hydrogen atoms per monomer unit replaced by fluorine) and polytetrafluoroethylene (PTFETeflon—all hydrogen replaced by fluorine) [18]. Increasing the levels of fluorine resulted in a reduction in shock speed, with PE having the highest and PTFE the lowest. However, due to a corresponding increase in density, the Hugoniot steepens with added fluorine. Interestingly, it was also noted that the release speed in PTFE was anomalously high, both here and in a previous article [23]. Although not stated explicitly in that work, it should be noted that PTFE undergoes a phase transition at approximately $0.5 \mathrm{GPa}$, see for example the work of Champion [24], Nagao et al. [25], Bourne et al. [26], Resnyansky et al. [27] and Rae et al. [28]. This has been identified as a phase II (a helical rotation over $13 \mathrm{CF}_{2}$ units in a hexagonal array) to phase III (a planar zig-zag confirmation in an orthorhombic lattice). Recovery experiments on PTFE by Brown et al. [29] showed that below the II-III phase transition decreases in crystallinity, Young's modulus and yield strength occurred, whilst above, the converse occurred, again indicating that significant microstructural changes occur at the phase transformation. Although not stated explicitly, it is possible therefore that these high release speeds may in fact be due to the high-pressure phase.

\section{Materials}

It is clear that even small alterations in the basic monomer unit can have a profound effect upon the shock response of even similar materials. Having observed the effects of side group size $[15,22]$ and degree of fluorination [18], we now 
turn our attention to the effects of a chlorine addition to the monomer. We have chosen two materials to act as a baseline; polyethylene $\left(\mathrm{CH}_{2}-\mathrm{CH}_{2}\right)_{\mathrm{n}}$ and PTFE $\left(\mathrm{CF}_{2}-\mathrm{CF}_{2}\right)_{\mathrm{n}}$, and replaced one hydrogen to produce polyvinylchloridePVC $\left(\mathrm{CH}_{2}-\mathrm{CHCl}\right)_{n}$ and one fluorine with chlorine to produce polychlorotrifluoroethylene-PCTFE/KelF-81 $\left(\mathrm{CF}_{2}-\right.$ $\mathrm{CFCl})_{\mathrm{n}}$. The basic conformation of the polymer molecules are displayed in Fig. 1.

The materials under investigation in this report were mostly obtained as commercial Stock of the Shelf (SoTS). However, some of the PTFE was also was from pedigreed sources, manufactured from pressed and sintered PTFE powders under the names DuPont PTFE 7A and DuPont PTFE 7C. Further details can be found in references [28, $30,31]$. The polyethylene investigated in this report was the high density form, studied in previous papers $[15,18$, $22,32]$. Relevant materials properties data are presented in Table 1.

\section{Experimental}

All shots were carried out using single stage gas guns (50 $\mathrm{mm}$ bore, $5 \mathrm{~m}$ long barrel and $51 \mathrm{~mm}$ bore, $6 \mathrm{~m}$ barrel) at Cranfield University [33]. Two sets of experiments were performed; equation of state experiments to investigate the

(a)

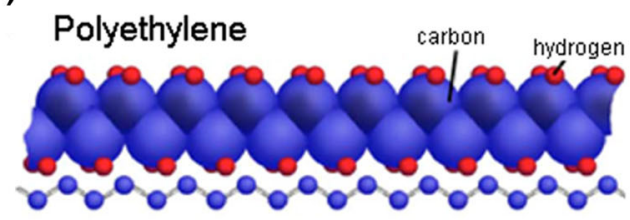

(b)

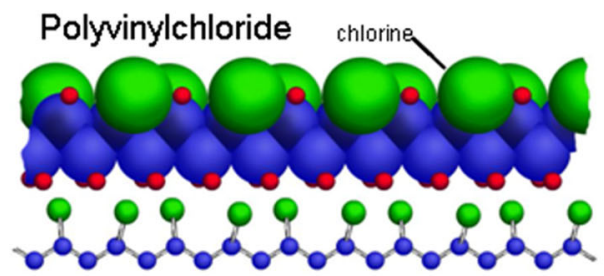

(c)

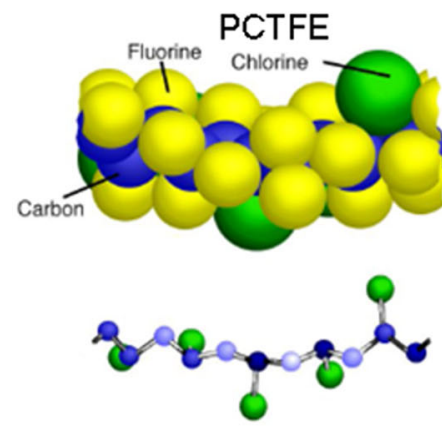

Hugoniot and release response and strength measurements to probe the variation of shear strength, both with impact stress and time behind the shock front. In the former, a manganin stress gauge (MicroMeasurments type LM-SS025CH-048) was embedded between plates (of known thickness) of the material of interest or supported on the rear surface with either a thick block of the target material or a $12 \mathrm{~mm}$ block of PMMA. This was to prevent releases from the rear of the target assembly interfering with releases from the rear of the flyer plate as it crossed the gauge location. A second gauge was supported on the front of the target assembly (the $0 \mathrm{~mm}$ position) with a $1 \mathrm{~mm}$ thick plate of either aluminium alloy 6061-T6 (Dural) or copper, and matched to the material of the flyer plate. In this way, both gauges would experience the Hugoniot stress generated by the impactor and through impedance matching the corresponding particle velocity, but also due to the temporal separation of the gauge traces $(\Delta t)$ along with the known physical separation of the gauges $\Delta w$ ), the shock velocity could be determined through $U_{\mathrm{S}}=\Delta w /$ $\Delta t$. Gauge calibrations were according to Rosenberg et al. [34] The second series of experiments was designed to investigate the strength response of these materials. $10 \mathrm{~mm}$ thick samples were sectioned in half and a manganin stress gauge (MicroMeasurements type J2 M-SS-580SF-025) was introduced $4 \mathrm{~mm}$ from the impact face $(2 \mathrm{~mm}$ in the (d)
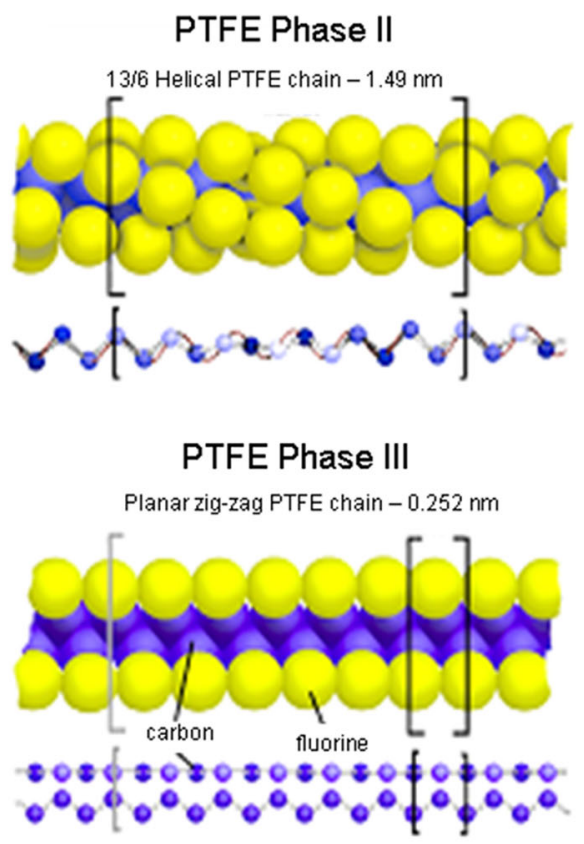

Fig. 1 The molecular structures of a polyethylene, b polyvinylchloride, c polychlorotrifluoroethylene, d polytetrafluoroethylene phase II, e polytetrafluoroethylene phase III 
Table 1 Properties of materials under investigation

\begin{tabular}{llllllllrr}
\hline & $\rho_{0}\left(\mathrm{~g} \mathrm{~cm}^{-3}\right)$ & $c_{\mathrm{L}}\left(\mathrm{mm} \mathrm{\mu s}^{-1}\right)$ & $c_{\mathrm{S}}\left(\mathrm{mm} \mathrm{\mu s}^{-1}\right)$ & $c_{\mathrm{B}}\left(\mathrm{mm} \mathrm{\mu s}{ }^{-1}\right)$ & $v$ & $K(\mathrm{GPa})$ & $\mu(\mathrm{GPa})$ & $T_{\mathrm{g}}\left({ }^{\circ} \mathrm{C}\right)$ & $T_{\mathrm{m}}\left({ }^{\circ} \mathrm{C}\right)$ \\
\hline PE & 0.95 & 2.36 & 1.01 & 2.05 & 0.388 & 3.99 & 0.98 & -100 & 110 \\
PVC & 1.42 & 2.28 & 1.11 & 1.89 & 0.345 & 5.07 & 1.74 & 87 \\
PTFE & 2.15 & 1.23 & 0.41 & 1.14 & 0.437 & 2.77 & 0.36 & -73 & 327 \\
PCTFE & 2.13 & 1.74 & 0.77 & 1.50 & 0.378 & 4.77 & 1.26 & 45 & 216 \\
\hline
\end{tabular}

$\rho_{O}$ ambient density, $c_{L}, c_{S}$ and $c_{B}$ longitudinal, shear and bulk sound speed, $v$ Poisson's ratio, $K$ bulk modulus, $\mu$ shear modulus, $T_{g}$ glass transition temperature, $T_{m}$ melting temperature

case of PCTFE) before reassembly, during which the sample was held in a special jig for a minimum of $12 \mathrm{~h}$ to allow the epoxy adhesive to cure. After this the impact face was lapped flat to within 5 optical fringes from a monochromatic light source. In the case of PCTFE, a second gauge was supported on the front of the target assembly with a $1 \mathrm{~mm}$ plate of either Dural or copper, matched to the flyer plate material. This was used to determine the longitudinal stress, otherwise it was calculated using the Hugoniot data measured in the previous experimental series. Lateral gauge data were analysed using the methods of Rosenberg et al. [35], taking into account the fact that the shape of the gauge will influence the result at low shock pressures. Schematic representations of the target assemblies are shown in Fig. 2.

The lateral stress gauge target was used to determine shear strength by measuring the lateral component of stress $\left(\sigma_{\mathrm{y}}\right)$. By assuming that the hydrostatic pressure during shock loading is the average of the three orthogonal components of stress,

$P=\frac{\sigma_{x}+\sigma_{y}+\sigma_{z}}{3}$

and that the two non-longitudinal stresses are equal, substituting Eq. 4 into Eq. 3 and re-arranging, shear strength can be determined thus,

$2 \tau=\sigma_{x}-\sigma_{y}$.

\section{Results}

A typical set of results from a longitudinal gauge experiment is presented in Fig. 3, in this case from PCTFE, with the back surface gauge supported with $12 \mathrm{~mm}$ of PMMA.

Note that there are two gauge traces. The first, labelled ' $0 \mathrm{~mm}$ ' comes from the Dural cover plate/PCTFE interface. The shape of the trace will be influenced by passage through ca. $1 \mathrm{~mm}$ of Dural, and as a consequence, it is relatively featureless, although its amplitude will give the longitudinal stress within the PCTFE, as dictated by the impact conditions, and by extension, the particle velocity, determined by standard impedance matching techniques. The second gauge, labelled 'Back Surface' is more revealing as the shock front has now travelled through ca. $10 \mathrm{~mm}$ of PCTFE, and hence its shape will have been modified by the properties of the PCTFE. The main feature to draw from here is the significantly reduced pulse width, indicating that PCTFE has an extremely high release wave speed, although this is explored further later in the text. The temporal spacing between traces, along with the physical spacing of the gauges themselves has been used to generate shock velocity [as indicated by the double arrow labelled $\Delta t$ (shock)], and the release speeds by the double arrow labelled $\Delta t$ (release).

The calculated shock velocities have been plotted against particle velocities and the results presented in Fig. 4a.
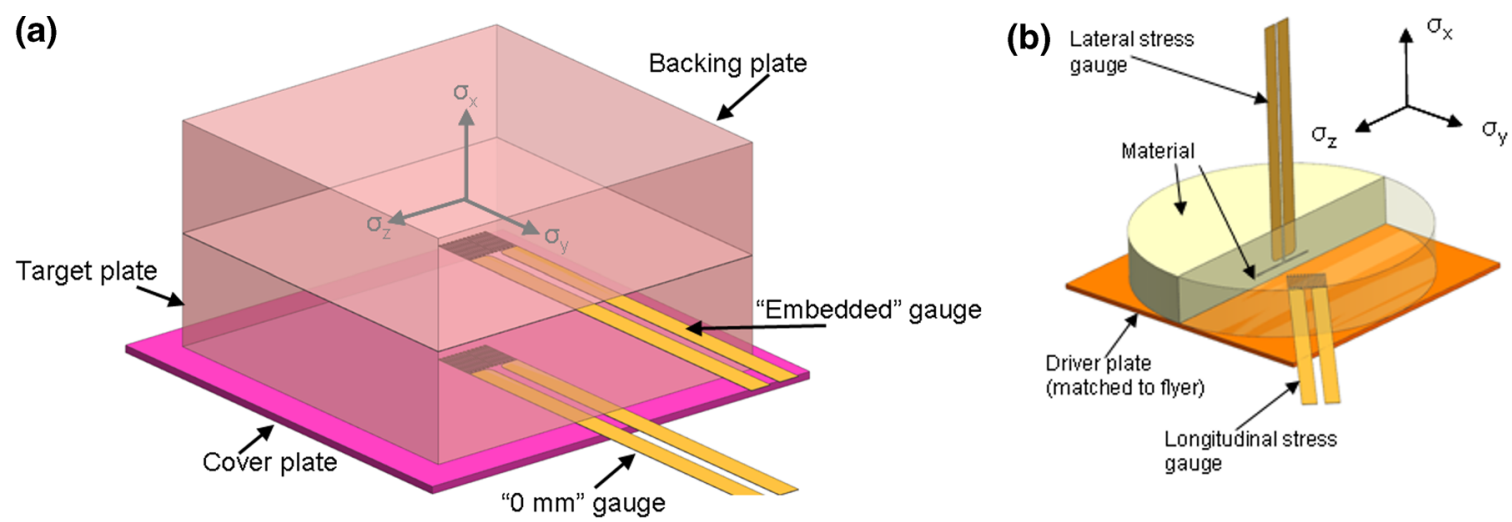

Fig. 2 Schematic diagrams of longitudinal and lateral stress gauge assemblies. a Longitudinal stress b lateral stress 


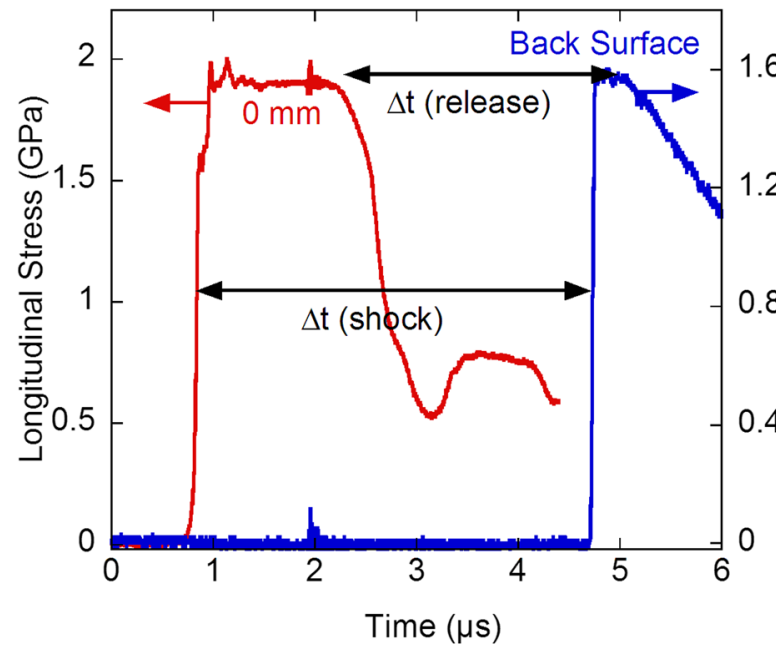

Fig. 3 Representative longitudinal stress gauge traces from a $10 \mathrm{~mm}$ PCTFE plate, with the back surface gauge supported with $12 \mathrm{~mm}$ of PMMA. The flyer plate was $5 \mathrm{~mm}$ of dural at $495 \mathrm{~m} \mathrm{~s}^{-1}$

All four polymers under investigation have a linear relationship between shock velocity and particle velocity, in common with many other materials [13]. Although a slight cusp can be observed in the Hugoniot of PTFE near $0.2 \mathrm{~mm} \mu \mathrm{s}^{-1}$ due to the phase II to III transition [27]. Simple linear fits according to Eq. 1 have been fitted, and the results summarised in Table 2 .

It should be noted that the values quoted in the above table are generated from a number of combined sources (see references). The values of $c_{0}$ and $S$ are simple empirical constants, although attempts have been made to give them physical significance; $c_{0}$ has been related to the bulk sound speed $\left(c_{\mathrm{B}}\right)$, whilst $S$ has been related to the first pressure derivative of bulk modulus [40]. In the case of simple metals such as copper, these assumptions have been seen to hold true [13]. However, with the polymers discussed in this report, it can be seen from Table 2 that $c_{0}$ clearly exceeds $c_{\mathrm{B}}$, indeed in the case of PTFE and PCTFE, it is greater than the longitudinal sound speed as well. In the case of PTFE, this might be due to the fact of a phase transformation at a stress of ca. 0.6 GPa [24]. However, in the case of PCTFE, no such phase transformation occurs, and as such another explanation is required. As all measurements in this investigation were made using stress gauges, the Hugoniot in terms of shock stress and particle velocity were also determined, and presented in Fig. 4b. Unlike the shock velocities, where clear differences can be seen, the Hugoniots are much more closely grouped, with PE having the shallowest curve, whilst the two fluorinated materials have a stiffer response, and PVC being placed somewhere between these two groups. Note that in Fig. 4b, the data has been
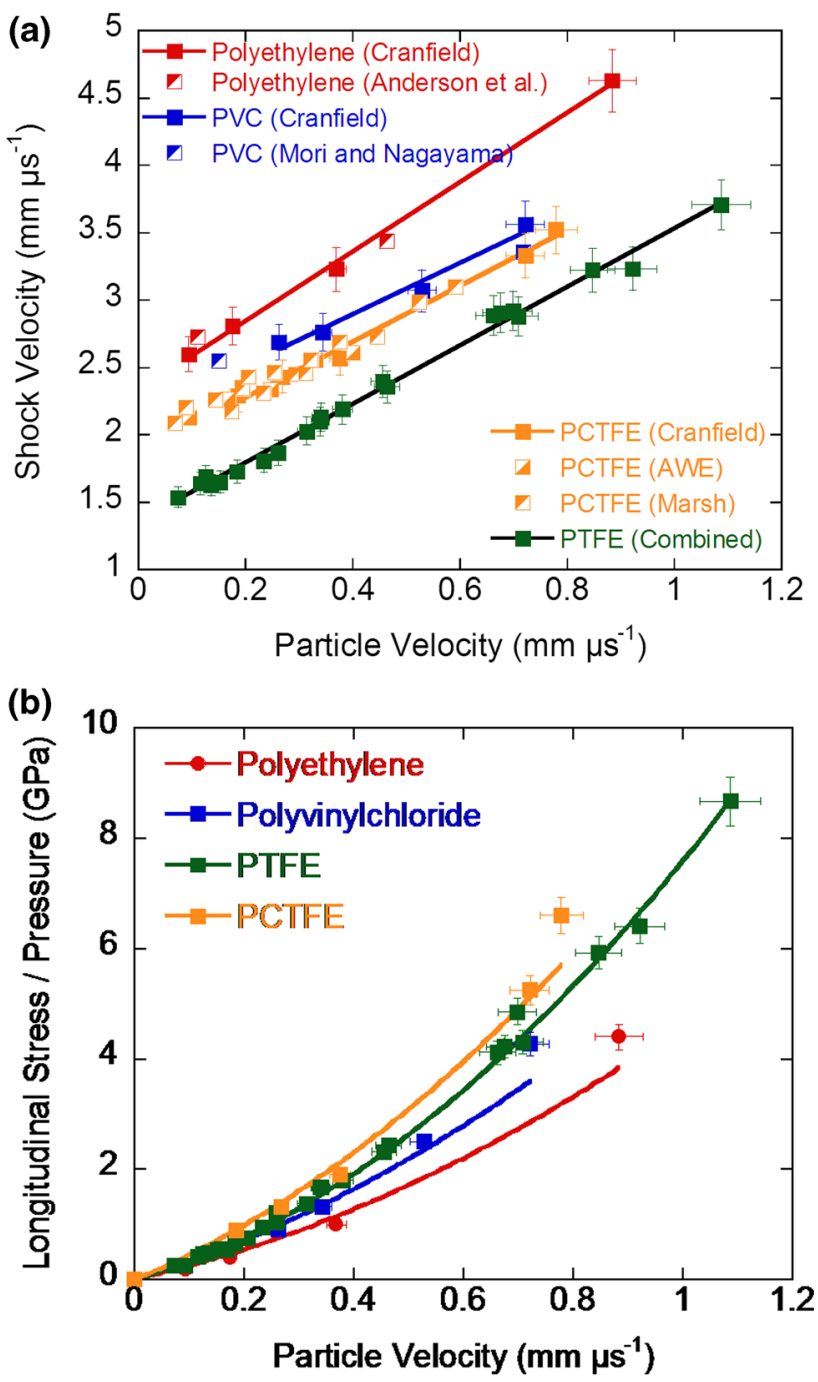

Fig. 4 Hugoniots of polymers under investigation. a Shock velocityparticle velocity. b Stress-particle velocity

fitted with Eq. 2, using the values of $c_{0}$ and $S$ quoted in Table 2. Note that in all materials (apart from PTFE), there is a divergence between measured longitudinal stress and calculated hydrodynamic pressure at higher particle velocities with stress being the greater. This is likely to be a first indication that these materials have increasing shear strength with shock stress (see Eq. 3). PTFE may also show this behaviour, but at higher stresses than explored in this paper.

From Fig. 3, the design of the target assemblies has allowed us to determine the velocity of the head of the release fan. These have been calculated using the known spacings of the gauges, taking into account that the material has been shock compressed, thus,

$U_{R}=\frac{\Delta w}{\Delta t \text { (release })}\left(1-\frac{u_{p}}{U_{S}}\right)$. 
Table 2 Shock relations for the polymers under investigation

\begin{tabular}{lllll}
\hline & $\rho_{0}\left(\mathrm{~g} \mathrm{~cm}^{-3}\right)$ & $c_{0}\left(\mathrm{~mm} \mathrm{\mu s}^{-1}\right)$ & $S$ & $c_{\mathrm{B}}\left(\mathrm{mm} \mathrm{\mu s}^{-1}\right)$ \\
\hline PE [14, 15, 18, 36] & 0.95 & 2.37 & 2.49 & 2.05 \\
PVC [37, 38] & 1.42 & 2.25 & 1.64 & 1.89 \\
PTFE [18, 24] & 2.15 & 1.36 & 2.17 & 1.14 \\
PCTFE [13, 39] & 2.13 & 1.93 & 1.94 & 1.50 \\
\hline
\end{tabular}

The results are presented in Fig. 5, both as basic release velocity (Fig. 5a) and normalised by the longitudinal sound speed (Fig. 5b).

It can be seen from Fig. 5a that the release velocity is linear in relation to particle velocity for all four materials under investigation, in common with a number of other polymers including polypropylene and polystyrene [15] and polycarbonate [41], and a glass fibre-epoxy composite
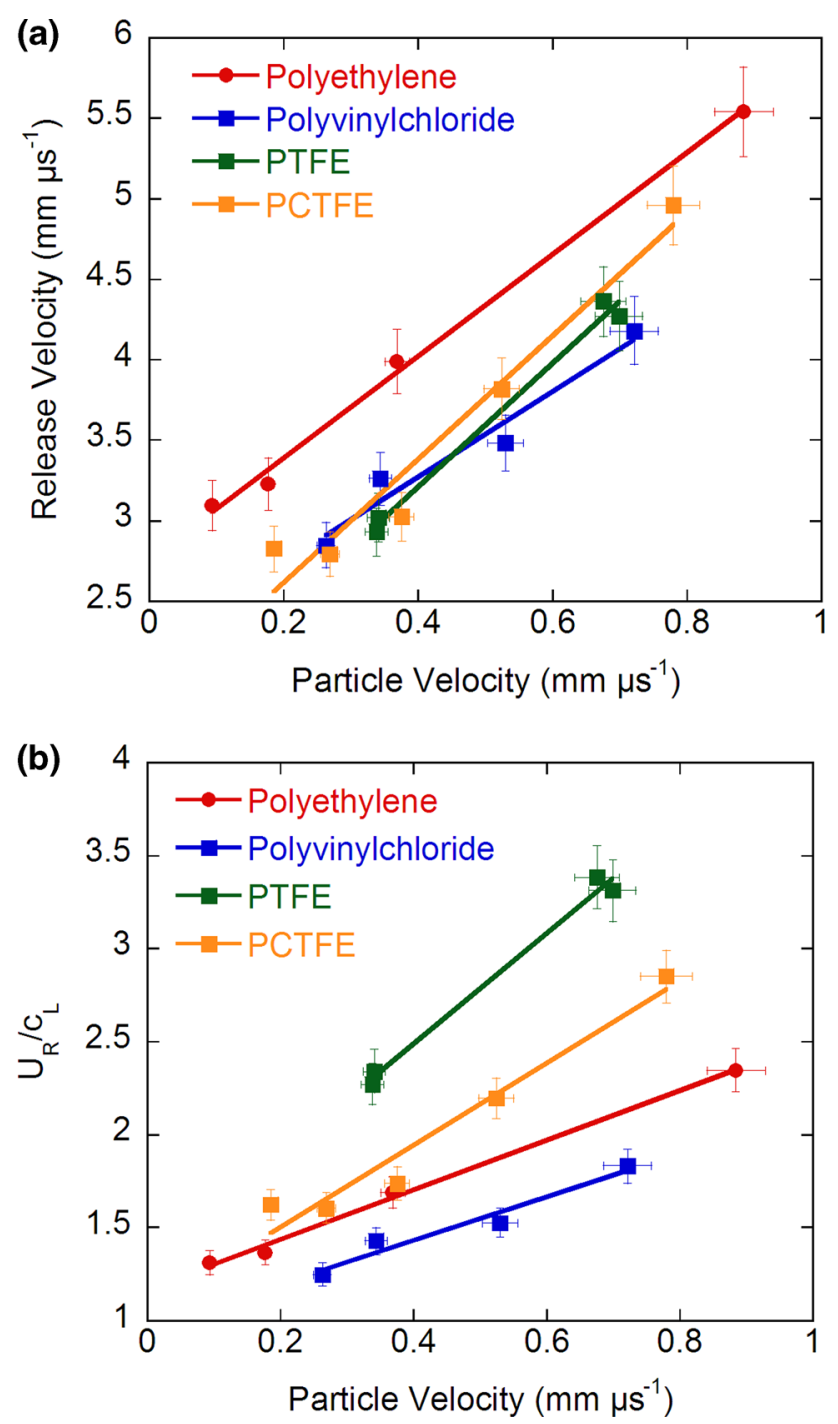

Fig. 5 Release response as a function of particle velocity. a Release velocity. b Normalised release velocity
[42]. However, given that the release response will be governed by the materials' properties and the shock response, it is difficult to identify trends from the basic release data. However, a previous article [18] made an attempt to normalise the release velocity by dividing by the shock velocity. This demonstrated that PTFE had an anomalously high release velocity when compared to either PE or PVDF, although no further explanation was given. In this work, we have modified this analysis slightly by normalising by the ambient longitudinal sound speed. The results are shown in Fig. 5b. Now a clearer picture emerges. PTFE has the highest relative release speeds, PE and PVC the lowest with PCTFE in the middle of the range. Further by assuming that relative release wave speed has a linear relationship with particle velocity of the form,

$\frac{U_{R}}{c_{L}}=A+B u_{p}$,

It is possible to draw tentative conclusions about the materials response. The results are summarised below in Table 3 .

In the majority of the materials in this investigation, the zero particle velocity intercept $A$ appears to trend toward a value of one, the exception being PTFE. We have used this analysis on the assumption that the head of the release wave travels at the longitudinal sound speed at the applied shock pressure. Therefore if true, it would be unsurprising if the release wave speeds trend back towards the ambient longitudinal sound speed. That PTFE does not is likely due to the phase II to III phase transition experienced on shock.

The final set of experiments was designed to probe the shear strength of these materials under shock loading. Representative lateral stress gauge traces from each material are presented in Fig. 6.

The basic form of these traces is a rapid rise to the peak lateral stress as the shock front crosses the gauge location, a near constant level of lateral stress behind the shock front

Table 3 Release characteristics

\begin{tabular}{lll}
\hline Material & $A\left({\left.\mathrm{~mm} \mu \mathrm{s}^{-1}\right)}^{B}\right.$ & $B$ \\
\hline Polyethylene & 1.17 & 1.34 \\
Polyvinylchloride & 0.97 & 1.17 \\
PTFE & 1.30 & 2.98 \\
PCTFE & 1.06 & 2.21 \\
\hline
\end{tabular}




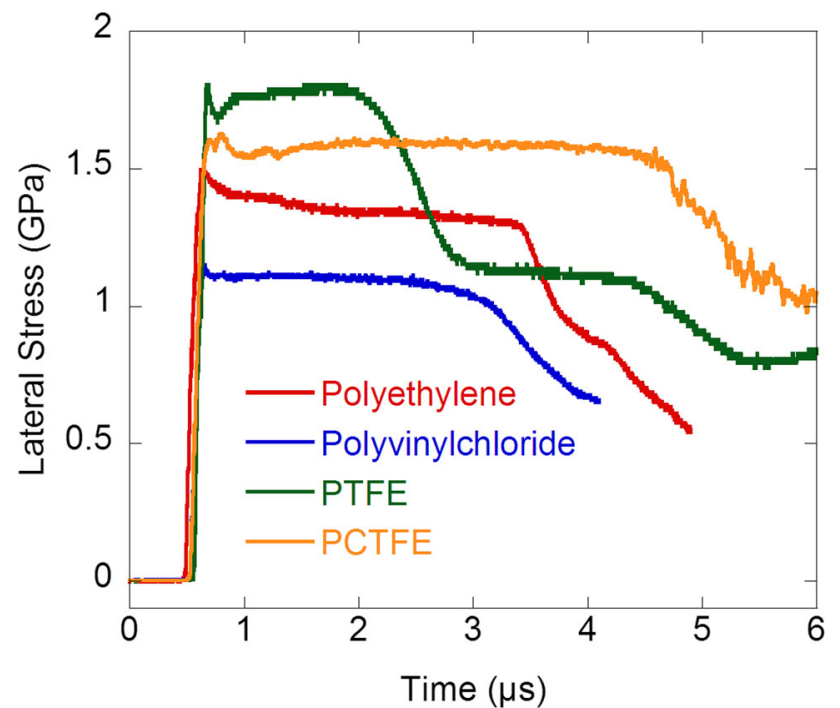

Fig. 6 Lateral stress gauge traces

before releases enter the gauge location and the material is returned to ambient conditions. The variation in pulse widths are explained by differing flyer plates; $10 \mathrm{~mm}$ Dural for PE and PVC, $10 \mathrm{~mm}$ copper for PCTFE and $5 \mathrm{~mm}$ copper for PTFE. Also observe that in some for PE and PVC, the lateral stress behind the shock front is not constant, but rather decreases slightly over time, indicating an increase in shear strength with time (Eq. 5). This has been observed previously in a number of other polymers, including PMMA [43, 44], polycarbonate [41] and PEEK [16]. This has been quantified and is presented below in Fig. 8. Before addressing this though, we consider the shock induced shear strength as a function of longitudinal stress, as presented in Fig. 7. These have been determined

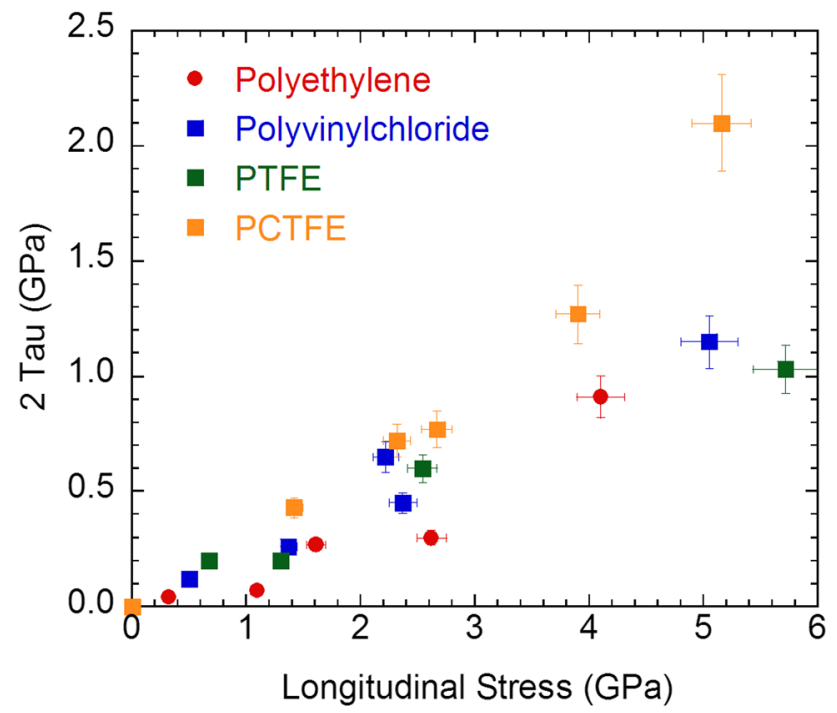

Fig. 7 Shear stress versus impact stress using Eq. 5, although the following qualification must be bourn in mind.

The lateral stress gauge traces from PTFE and PCTFE are essentially flat behind the shock front (until releases enter the gauge location), hence the calculation of shear strength from the known longitudinal stress is trivial. The situation with PE and PVC is complicated by the fact that lateral stress behind the shock front decreases, thus indicating an evolving (increasing) shear strength with time. Therefore, the shear strength for these two materials presented in Fig. 7 have been determined immediately behind the shock front. These results indicate that there is little difference between the shear strengths of PE, PVC and PTFE, and their variation with imposed shock stress. At higher impact stresses, there does seem to be a strengthening in PCTFE, suggesting that modification of a predominately fluorinated polymer has a greater effect on strength than similar modifications on a simple polyethylene based polymer.

The final point we wish to discuss is the rate at which lateral stress changes behind the shock front, giving an indication of the kinetics of shock induced material deformation in these materials. The results are presented in Fig. 8.

As indicated by the lateral stress traces shown in Fig. 6, the two fluorinated-materials have a largely flat response, indicating zero change behind the shock front. In contrast, PE and PVC both appear to harden behind the shock front, with the level of that hardening increasing with imposed shock stress. Further, it would appear that the degree of that hardening is near identical between these two materials. In previous works [22, 41] where this behaviour has been observed, it has been suggested that this be due to steric

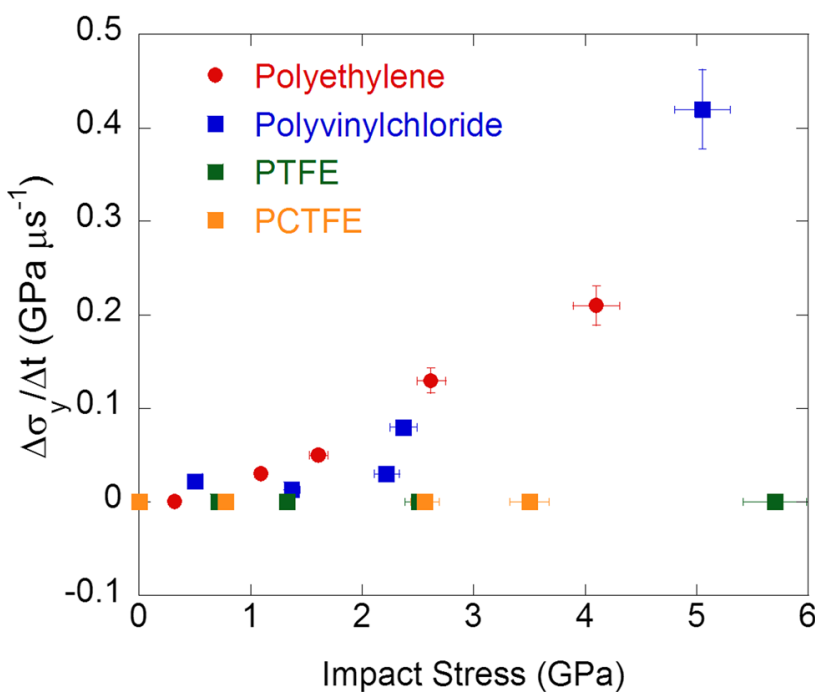

Fig. 8 Lateral stress variation with impact stress 
interference between adjacent polymer chains that is responsible. In the case of fluorinated polymers, it was proposed that the high electronegativity of the fluorine atoms, combined with the sheathing effect of those atoms around the carbon backbone allowed much easier passage of the polymer chains past each other, thus resulting in a flatter lateral stress response [18].

\section{Discussion}

The shock response of the four common polymers studied in this investigation are in the main affected by two factors. The first is an electronic component due to repulsion between the constituent atoms as they approach each other during shock compression. It would be expected that this would be greatest in PTFE, where the carbon backbone is sheathed by large (compared to hydrogen), strongly electronegative fluorine atoms. The second is due to the physical interaction between adjacent polymer chains due to their shape, in other words chain tangling or tacticity. This would be expected to be greatest in a molecule where a side group off a carbon atom disrupts the otherwise regular ordering of the basic monomer unit, thus tangling effects would be expected to be greater in PVC than PE, and greater in PCTFE than PTFE. However, these effects do not act in isolation, but rather in combination, and it is the intention of this paper to indicate how these factors affect the overall shock response.

The first point to consider is the equation of state, as shown in Fig. 4 and Table 2. For the sake of clarity, we reproduce the values of $S$ along with the ambient conditions bulk modulus in Table 4 , ordered by decreasing value of $S$.

Although the shock parameters $c_{0}$ and $S$ are empirical constants, $c_{0}$ has been related (in simple metals at least) to the bulk sound speed [13,40], whilst $S$ has been linked to the first pressure derivative of bulk modulus [40]. From this, compressibility decreases from polyethylene, to PTFE to PCTFE with PVC having the least compressibility. If we take PE as the base line polymer, it would appear that any degree of modification to the polymer chain has a stiffening effect. Therefore PTFE is stiffer than PE due to the repulsive effect of the highly electronegative fluorine

Table 4 Values of $S$ and bulk modulus

\begin{tabular}{lll}
\hline & $S$ & $K(\mathrm{GPa})$ \\
\hline Polyethylene & 2.49 & 3.99 \\
Polytetrafluoroethylene & 2.17 & 2.77 \\
Polychlorotrifluoroethylene & 1.94 & 4.77 \\
polyvinylchloride & 1.64 & 5.07 \\
\hline
\end{tabular}

atoms resisting the compression of adjacent polymer chains. The chemically similar PCTFE has less compressibility than PTFE, even though the additional chlorine atom replacing a single fluorine atom will have minimal changes to the repulsion between chains due to the similarities between the electronegativities between chlorine and fluorine. Therefore the stiffening effect is most likely the result in the larger chlorine atom giving the polymer chain some degree of tacticity, allowing a small degree of increased resistance between polymer chains. The stiffest material of all is PVC, with an $S$ of 1.64. Given the hypothesis given above, one would expect that the addition of a single chlorine atom to the basic PE chain would only have a small degree of repulsion between chlorine atoms, and even with the additional tangling effects due to the addition of the larger (compared to hydrogen) atoms, the over all stiffening effect would not be great. However, an additional effect lies in the alternating $\mathrm{CH}_{2}-\mathrm{CHCl}$ units in the polymer chain. This will result in local changes in charge density, which will effect how the polymer chains interact. In a previous paper [18], a similar effect was noted between PTFE and polyvinylidene difluoride (PVDF) where alternating $\mathrm{CH}_{2}-\mathrm{CF}_{2}$ units were shown to significantly affect the shock response. Finally we would also point out that although the overall ranking of $S$ appears to agree with that of the ambient bulk modulus, the local trend between PE and PTFE is reversed. Under ambient conditions, PTFE has a lower bulk modulus than PE, suggesting that it is more compressible. However, it should be noted that PTFE undergoes a pressure induced phase change at ca. $0.5 \mathrm{GPa}[24,45]$, and hence if the properties of PTFE in its ambient phase II state and high pressure phase III state are significantly different, it would explain this variation of behaviour between bulk modulus and $S$.

Examination of the release behaviour (Fig. 5; Table 3) also reveals the interplay between repulsion between polymer chains and chain tangling. From Fig. 5b, it can be seen that the ordering of relative release velocities (fastest to slowest) is PTFE, PCTFE, PE with PVC being the slowest. The same ordering can also be seen for slope of the $U_{\mathrm{R}} / c_{\mathrm{L}-} u_{\mathrm{p}}$ curve, $B$. In the case of PTFE, the high electronegativity of the sheathing fluorine atoms will drive the adjacent polymer chains apart on release resulting in the high release speeds observed in Fig. 5a. A similar behaviour will affect PCTFE, where one would expect a high release speed. From Fig. $5 \mathrm{~b}$ and Table 3, it can be seen that this is the case, although the overall release speed is less than that of PTFE. We believe that this is due to the influence of the replacement chlorine atom conveying a small degree of tacticity, allowing adjacent chains a small degree of resistance against electrostatic repulsion. With $\mathrm{PE}$, the degree of electrostatic repulsion will be minimal and hence release speeds will be dominated by chain 
tangling. As a consequence, the release speeds will be significantly lower as the chains resist coming apart. Finally, PVC has the lowest release speed and value of $B$ of all four materials in this investigation. We believe two factors are in effect here. Firstly, the large chlorine atom (replacing a hydrogen) will increase the degree of chain tacticity compared to PE. Secondly, the highly electronegative chlorine atom will result in a variation of charge density along the PVC chain that will allow for additional localised attractive forces between adjacent chains, which will further reduce the release velocity.

The final side to this investigation was to examine the effects of additional chlorine atoms on the shock induced strength. As with many shock-loaded materials, there are two components to this response; a variation with time (behind the shock front) and a variation with pressure. Looking at the lateral stress gauge profiles in Fig. 6 and the quantified changes in lateral stress with respect to time (Fig. 8), it can be seen that the four materials under investigation can be split into two groups. PE and PVC show a reduction in lateral stress, and hence an increase in shear strength behind the shock front. From Fig. 8, it would appear that these changes with respect to impact stress are near identical. In contrast, both PTFE and PCTFE show a near constant lateral stress and hence shear strength behind the shock front, in common with a number of other fluorinated polymers such as KelF-800 (a bi-polymer between chlorotrifluoroethylene and vinylidene difluoride) [46] and Viton-B (a tri-polymer of tetrafluoroethylene, hexafluoropropylene and vinylidene difluoride) [47]. In the case of PTFE, we have suggested that the sheathing of the carbon backbone by large fluorine atoms has two effects; effectively making the polymer chain smoother, thus allowing easier movement between adjacent polymer chains, and high degree of electrostatic repulsion between chains due to the presence of the same strongly electronegative fluorine atoms. As a consequence, the chains will move relatively easily past each other, hence there will be little change in strength with time. In contrast, the relatively open nature of the PE molecule allows greater physical interaction between adjacent polymer chains (in other words tangling) and hence as tangling increases, the stresses required to move them together will increase with time. A similar response will occur in PVC where the addition of a single chlorine atom to the basic polyethylene monomer will enhance the propensity for adjacent polymer chains to tangle. From Figs. 6 and 8, it can be seen that PCTFE behaves in a similar way to PTFE. It would appear that any changes in tacticity due to the replacement of a fluorine atom by a chlorine atom are minimal, most likely due to the still strong electronegativity of the additional chlorine atom. However, the fact that other fluorinated polymers (KelF-800 and Viton-B) also display this response, even though they have dangling side groups (chlorine atoms and trifluoromethyl) and alternating $\mathrm{CF}_{2}-$ $\mathrm{CH}_{2}$ groups (Kelf-800 and Viton-B) would suggest that these materials should display a degree of tacticity resulting in a hardening response behind the shock front. The fact that they do not is an indication that the electrostatic repulsion between chains (when present) has a much stronger influence than chain tangling, operating at a greater inter chain separation that prevents physical interaction between chains from coming into effect. Finally, from Fig. 7 it can be seen that the strength of all four polymers under investigation increase with shock stress. Although there is a degree of scatter within the data, it would appear that the ordering (lowest strength first) is PE, PTFE, PVC and finally PCTFE with the highest strength. Therefore, the replacement of hydrogen with fluorine atoms has a strengthening effect due to the large increase in electrostatic repulsion between PE and PTFE, whilst the increase between PE and PVC is more likely to be due to an increase in polymer chain tacticity due to the addition of a single chlorine atom. PCTFE has the highest strength of all, suggesting that there is a cumulative effect between electrostatic repulsion and tacticity. Although Fig. 8 shows no evidence of hardening behind the shock front in PCTFE, the significant increase in overall shear strength at high stresses (compared to the other three materials under investigation) may be a first indication that chain tangling may be having a more significant role in the shock induced shear strength of PCTFE.

We therefore propose that the strength of polymers has two main components. Firstly, there will be an electrostatic repulsion between adjacent chains, the strength of which will depend on the precise chemistry of those chains. In the case of (mostly) hydrocarbon based polymer chains, this will be a relatively weak force that is readily overcome by the compression supplied by the shock. Conversely, where there are a large number of halogen atoms (principally fluorine) surrounding the basic carbon backbone, that interchain repulsive force will be much greater, and in many materials will actually dominate the materials mechanical response to shock loading. Secondly, there will be an effect due to the shape of the polymer, which allows adjacent chains to tangle as the shock pressure brings them closer together (tacticity). This will be more prevalent in materials with an open chain morphology (PE) or dangling side groups (PVC). This behaviour will operate at lesser distances than the electrostatic repulsion discussed above, and will only come into operation when this first force has been overcome. We hypothesis that at shock stresses greater than those employed in this study, heavily fluorinated polymers such as PTFE may begin to show hardening behaviour behind the shock front, similar to that displayed by PE at lower shock stresses. That PCTFE, of the four 
materials discussed in this report, displays the greatest shear strength at the highest shock stresses could be a first indication of chain tacticity having a greater role in its shock response.

\section{Conclusions}

The effects of replacing either a hydrogen or fluorine atom in polyethylene and polytetrafluorethylene (to create PVC and PCTFE respectively) on the shock response has been investigated in terms of the Hugoniot, release velocities and shear strengths. The results from these shots have led us to suggest that there are two main factors affecting the shock response of simple polymers; an electrostatic repulsive force between adjacent polymer chains and a physical interaction due to tangling (chain tacticity). The former is dominant when the polymer chain is surrounded by highly electronegative atoms such as fluorine (PTFE) and/or chlorine (PCTFE). That these large (compared to hydrogen) atoms also has a sheathing effect upon the polymer chain, resulting in an over all smoother shape that allows easier passage of the polymer chains past each other during shock loading. Under such circumstances, this will result in a comparatively low shock speed, high release speed and a lack of hardening behind the shock front. In contrast, where inter chain tangling becomes dominant, the shock speed increases, the release speed reduces and hardening behind the shock front becomes significant. The addition of a single chlorine atom on either the PE (forming PVC) or PTFE chain (forming PCTFE) results in significant modifications of the basic shock response. PVC has a higher shock speed, lower release speed and reduced compressibility compared to PE. We believe that this is in part due to the large single chlorine atom on the base monomer unit increasing the likelihood of chain tacticity when compared to PE. However, we also suggest that the same chlorine atom will also result in localised changes in charge density along the chain, which could modify interchain interactions. In the case of PCTFE, the role of the additional chlorine atom also appears to result in an increase in shock-speed, reduction in release speed and an increase in shear strength at higher impact stresses. We therefore suggest that the shock response of these polymers is controlled by the interplay of these factors. As the shock pressure compresses the material, the initial behaviour is due to the electrostatic repulsion between chains. As pressure increases, the polymer chains move closer together and hence physical interaction between those chains, in the main due to tacticity but also due to charge distributions along the chains will become increasingly important. In heavily fluorinated materials such as PTFE and PCTFE, it is the repulsive forces that dominate. In contrast, in (predominantly) hydrocarbon polymers, the repulsive forces are weak, and hence inter chain tacticity becomes the dominant factor controlling shock response. It is likely that the hardening response behind the shock front displayed by PE and PVC would also be exhibited by PTFE and PCTFE at higher impact stresses, although at present these would be higher than the pressures investigated here.

Acknowledgments The authors would like to thank the staff of Cranfield University and Los Alamos National Laboratory for their technical assistance in this programme. (C) British Crown Owned Copyright 2016/AWE. Published with permission of the Controller of Her Britannic Majesty's Stationery Office. "This document is of United Kingdom origin and contains proprietary information which is the property of the Secretary of State for Defence. It is furnished in confidence and may not be copied, used or disclosed in whole or in part without prior written consent of Defence Intellectual Property Rights DGDCDIPR-PL-Ministry of Defence, Abbey Wood, Bristol, BS348JH, England.”

\section{References}

1. Milne A, Longbottom A, Bourne N, Millett J (2007) On the unreacted Hugoniots of three plastic bonded explosives. Propellants, Explos, Pyrotech 32:68-72

2. John HJ Jr, Hudson FE, Robbs R (1998) High strain rate testing of AP/Al/HTPB solid propellants. In: Schmidt SC, Dandekar DP, Forbes JW (eds) Shock compression of condensed matter 1997. American Institute of Physics, Woodbury, pp 603-608

3. Sutherland GT, Forbes JW, Lemar ER, Ashwell KD, Baker RN (1994) Multiple stress-time profiles in a RDX/AP/Al/HTPB plastic bonded explosive. In: Schmidt SC, Shaner JW, Samara GW, Ross M (eds) High pressure science and technology 1993. American Institute of Physics, New York, pp 1381-1384

4. Hoffman DM (2003) Dynamic mechanical signatures of Viton A and plastic bonded explosives based on this polymer. Polymer Eng Sci 43:139-155

5. Munson DE, Boade RR, Schuler KW (1978) Stress wave propagation in $\mathrm{Al}_{2} \mathrm{O}_{3}$-epoxy mixtures. J Appl Phys 49:4797-4807

6. Setchell RE, Anderson MU (2005) Shock-compression response of an alumina-filled epoxy. J Appl Phys 97:083518

7. Dandekar DP, Hall CA, Chhabildas LC, Reinhart WD (2003) Shock response of a glass-fiber-reinforced polymer composite. Compos Struct 61:51-59

8. Buland P, Dalbera J and Lafolie R (1995) Seismic behaviour of non-sliding laminated neoprene bearings subjected to large distortion. In: Sum (ed) 10th European conference on Earthquake Engineering. Balkema, Rotterdam, pp 2029-2032

9. Barker LM, Hollenbach RE (1970) Shock-wave studies of PMMA, fused silica, and sapphire. J Appl Phys 41:4208-4226

10. Munson DE, May RP (1972) Dynamically determined highpressure-compressibilities of three epoxy resin systems. J Appl Phys 43:962-971

11. Millett JCF, Bourne NK, Barnes NR (2002) The behaviour of an epoxy resin under one-dimensional shock loading. J Appl Phys 92:6590-6594

12. Appleby-Thomas GJ, Hazell PJ, Stennett C (2009) The variation in lateral and longitudinal stress gauge response within an RTM 6 epoxy resin under one-dimensional shock loading. J Mater Sci 44:6187-6198

13. Marsh SP (1980) LASL shock Hugoniot data. University of California Press, Los Angeles 
14. Carter WJ, Marsh SP (1995) Hugoniot equation of state of polymers, LA-12006-MS. Los Alamos National Laboratory, Los Alamos, NM

15. Millett JCF, Bourne NK (2004) The equation of state of three simple polymers. J Phys D Appl Phys 37:2901-2907

16. Millett JCF, Bourne NK, Gray GT III (2004) The response of polyether ether ketone to one-dimensional shock loading. J Phys D Appl Phys 37:942-947

17. Millett JCF, Bourne NK (2006) The shock induced equation of state and shear strength of polyvinylidene difluoride. J Phys IV 134:719-724

18. Bourne NK, Millett JCF, Brown EN, Gray GT III (2007) Effect of halogenation on the shock properties of semicrystalline thermoplastics. J Appl Phys 102:063510

19. Millett JCF, Bourne NK, Akhavan J, Milne AM (2005) The response of soda-lime glass-hydroxyterminated polybutadiene composites to shock loading. J Appl Phys 97:043524

20. Bourne NK, Milne AM (2004) Shock to detonation transition in a plastic bonded explosive. J Appl Phys 95:2379-2385

21. Brown EN, Rae PJ, Dattelbaum DM, Clausen B, Brown DW (2008) In-situ measurement of crystalline lattice strains in polytetrafluoroethylene. Exp Mech 48:119-131

22. Bourne NK, Millett JCF (2008) Tacticity in shocked polymer hydrocarbons. J Mater Sci 43:185-189

23. Bourne NK, Gray GT III (2003) Equation of state of polytetrafluroethylene. J Appl Phys 93:8966-8969

24. Champion AR (1971) Shock compression of teflon from 2.5 to 25 kbar-evidence for a shock induced transition. J Appl Phys 42:5546-5550

25. Nagao H, Matsuda A, Nakamura KG, Kondo K (2003) Nanosecond time-resolved Raman spectroscopy on phase transition of polytetrafluroethylene under laser-driven shock compression. Appl Phys Lett 83:249-250

26. Bourne NK, Brown EN, Millett JCF, Gray GT III (2008) Shock, release and Taylor impact of the semicrystalline thermoplastic polytetrafluoroethylene. J Appl Phys 103:074902

27. Resnyansky AD, Bourne NK, Millett JCF, Brown EN (2011) Constitutive modelling of shock response of polytetrafluoroethylene. J Appl Phys 110:033530

28. Rae PJ, Brown EN, Clements BE, Dattelbaum DM (2005) Pressure-induced phase change in poly(tetrafluoroethylene) at modest impact velocities. J Appl Phys 98:063521

29. Brown EN, Trujillo CP, Gray GT III, Rae PJ, Bourne NK (2007) Soft recovery of polytetrafluoroethylene shocked through the crystalline phase II-III transition. J Appl Phys 101:024916

30. Brown EN, Rae PJ, Gray GT III (2006) The influence of temperature and strain rate on the tensile and compressive constitutive response for four fluoropolymers. J Phys IV France 134:935-940

31. Brown EN, Rae PJ, Orler EB, Gray GT III, Dattelbaum DM (2006) The effect of crystallinity on the fracuture of polytetrafluoroethylene (PTFE). Mater Sci Eng C 26:1338-1343
32. Bourne NK, Millett JCF (2008) On the influence of chain morphology on the shock response of three thermoplastics. Metall Mater Trans A 39:266-271

33. Bourne NK (2003) A $50 \mathrm{~mm}$ bore gun for dynamic loading of materials and structures. Meas Sci Technol 14:273-278

34. Rosenberg Z, Yaziv D, Partom Y (1980) Calibration of foil-like manganin gauges in planar shock wave experiments. J Appl Phys 51:3702-3705

35. Rosenberg Z, Bourne NK, Millett JCF (2007) On the effect of manganin geometries upon their response to lateral stress. Meas Sci Technol 18:1843-1847

36. Anderson MU (1992) Response of the polymers Kel-F, polysulfone, high density polyethylene and PMMA to shock loading and release from 0.3 to $2.4 \mathrm{GPa}$. In: Schmidt SC, Dick RD, Forbes JW, Tasker DG (eds) Shock compression of condensed matter1991. Elsevier, Amsterdam, pp 875-878

37. Butler S, Millett JCF, Bourne NK (2008) Shock induced equation of state of polyvinylchloride. In: Furnish MD, Elert ML (eds) Shock compression of condensed matter-2007. AIP Press, Melville, pp 695-698

38. Mori Y, Nagayama K (1999) Shock Hugoniot curves for several polymeric materials in $0.5 \mathrm{GPa}$ shock stress. SPIE 3516:241-245

39. Millett JCF, Lowe MR, Appleby-Thomas G, Roberts A (2016) The mechanical and optical response of polychlorotrifluoroethylene to one-dimensional shock loading. Metall Mater Trans A 47A:697-705

40. Davison L, Graham RA (1979) Shock compression of solids. Phys Rep 55:255-379

41. Millett JCF, Bourne NK (2006) Shock and release of polycarbonate under one-dimensional shock loading. J Mater Sci 41: $1683-1690$

42. Millett JCF, Meziere YJE, Bourne NK (2007) The response to shock loading of a glass-fibre-epoxy composite: effects of fibre orientation to the loading axis. J Phys D: Appl Phys 40:53585365

43. Millett JCF, Bourne NK (2000) The deviatoric response of polymethylmethacrylate to one-dimensional shock loading. J Appl Phys 88:7037-7040

44. Gupta SC, Gupta YM (1985) Piezoresitive response of longitudinally and laterally orientated ytterbium folis subjected to impact and quasi-static loading. J Appl Phys 57:2464-2473

45. Brown EN, Dattelbaum DM, Brown DW, Rae PJ, Clausen B (2007) A new strain path to inducing phase transitions in semicrystalline polymers. Polymer 48:2531-2536

46. Bourne NK, Gray GT III (2005) Dynamic response of binders; teflon, estane and Kel-F-800. J Appl Phys 98:123505

47. Millett JCF, Brown EN, Bourne NK, Gray GT III (2008) The behaviour of a fluorinated polymer during one-dimensional shock loading. In: SEM IX international congress and exposition on experimental and applied mechanics, Orlando, FL, p 123 